IRRITABLE BOWEL SYNDROME

\title{
Sexual and physical abuse are not associated with rectal hypersensitivity in patients with irritable bowel syndrome
}

\author{
Y Ringel, W E Whitehead, B B Toner, N E Diamant, Y Hu, H Jia, S I Bangdiwala, D A Drossman
}

Gut 2004;53:838-842. doi: 10.1136/gut.2003.021725

See end of article for authors' affiliations

Correspondence to: Dr Y Ringel, Division of Gastroenterology and Hepatology, 1140 Biolnformatics Bldg, CB\#7080, 130 Mason Farm Rd, University of North Carolina at Chapel Hill, Chapel Hill, NC 27599-7080, USA ringel@med.unc.edu

Accepted for publication 23 December 2003
Background: Patients with irritable bowel syndrome (IBS) have reduced pain thresholds for rectal distension. In addition, the prevalence of sexual/physical abuse in referred IBS patients is high and is associated with greater pain reporting, poorer health status, and poorer outcome. This lead to a hypothesis that abuse history may sensitise patients to report pain at a lower threshold.

Aim: To compare rectal pain thresholds in women with IBS who had a history of severe abuse to IBS women with no history of abuse.

Methods: We studied 74 IBS patients with a history of severe physical and/or sexual abuse and 85 patients with no history of abuse. Abuse history was assessed by a previously validated self-report abuse screening questionnaire. Rectal sensory thresholds were assessed using an electronic barostat and determined by the ascending method of limit (AML) and by the tracking technique.

Results: IBS patients with a history of severe abuse had significantly higher rectal pain thresholds, as measured by $A M L(F(1,111)=6.06 ; p=0.015)$ and the tracking technique $(F(1,109)=5.21 ; p=0.024)$. Patients with a history of severe abuse also reported a significantly higher threshold for urgency to defecate $(F(1,113)=11.23 ; p=.001)$.

Conclusion: Severe sexual/physical abuse is associated with higher urge and pain thresholds for rectal distension in IBS patients. This suggests that the greater pain reporting and poorer health status in IBS patients with abuse history are not related to increased rectal pain sensitivity. Further studies are needed to determine the causes of these findings.
$\mathrm{R}$ educed pain thresholds for rectal sensation and pain have been proposed as a possible mechanism in the pathophysiology of irritable bowel syndrome (IBS). ${ }^{12}$ When compared with controls, IBS patients experience urge to defecate and report pain sensations at lower rectal volume/ pressure. $^{23}$ This phenomenon of visceral hypersensitivity has also been described in other parts of the gastrointestinal tract (for example, oesophagus, stomach, small intestine, and colon), supporting its role in the pathogenesis of the disorder..$^{3-5}$ Moreover, there are data to suggest that changes in rectal sensation thresholds correlate with changes in clinical pain symptoms. ${ }^{16}$ Nevertheless, our current understanding of IBS suggests a major influence for various psychosocial factors (for example, abuse history, life stressors) in addition to physiological factors (for example, rectal hypersensitivity) on the clinical presentation of the disorder (that is, the biopsychosocial model of IBS). ${ }^{78}$

Psychosocial factors, including a history of sexual and/or physical abuse, are associated with IBS and contribute to its adverse outcome. ${ }^{9-12}$ Epidemiological studies show a high prevalence of abuse history among female patients in referral gastrointestinal clinics regardless of the diagnosis ${ }^{13}$ and particularly in referred patients with more severe IBS. ${ }^{9}{ }^{13-16}$ Sexual and/or physical abuse is reported in up to $50 \%$ of IBS patients seen in this setting, which is a higher prevalence than seen in the general population or in patients with organic gastrointestinal disorders. ${ }^{15}$ Furthermore, we have previously reported that a history of abuse is associated with greater pain reporting, greater psychological distress, poorer health status, and poorer outcome in patients with IBS. ${ }^{9}$

The association of a history of sexual abuse with both the diagnosis of IBS and its poorer outcome has suggested to some that abuse history may sensitise patients to report pain at a lower threshold. ${ }^{8}{ }^{17}$ The only study that examined this issue $^{18}$ found no evidence for lower pain thresholds to be associated with abuse. However, this earlier study was done in a relatively small sample, which may have led to a type II statistical error. Given these clinical observations, it remains unclear whether abuse history leads to greater pain reports and poorer health status because of peripheral effects (for example, peripheral visceral hypersensitivity/reduced sensation thresholds) or central effects (for example, central hypervigilancy and disinhibition of pain modulation).

The aim of the present study was to compare rectal pain thresholds in a large group of women with IBS, who had a history of severe sexual abuse, to a large group of IBS women with no history of abuse. Lower pain thresholds in patients with abuse history would suggest a peripheral and/or central mechanism, while a normal or increased pain threshold might suggest a central effect of abuse on pain perception.

\section{METHODS}

\section{Subjects}

A total of 278 female patients with IBS who were enrolled in a multicentre clinical trial at the University of North Carolina at Chapel Hill and at the University of Toronto, were assessed. The analyses reported here are limited to 74 IBS patients who met the criteria for severe abuse (see below) and 85 patients with no history of abuse. All patients had moderate or severe IBS, as determined by the functional bowel disorder severity index. ${ }^{19}$ Participants were recruited by physician referrals, self-referrals, and advertising in the community. Subjects with a major psychiatric diagnosis (for example, bipolar disorder) or who were taking antidepressant medication were excluded from the study. The study was approved by the Institutional Review Board of the

Abbreviations: Abbreviations: IBS, irritable bowel syndrome; AML, ascending method of limit; $\Pi$, tracking technique; VAS, visual analogue scale 
University of North Carolina (UNC), and all subjects gave informed consent.

\section{Assessment of abuse history/psychosocial factors}

Abuse history was assessed by a self-report abuse screening questionnaire that has been validated by interview. ${ }^{20}$ On the basis of responses to this questionnaire, severe abuse was defined as having been raped; or often hit, kicked, or beaten; or often having experienced threats to one's life, either as a child or an adult. No abuse was defined as no rape, no unwanted touching of genitalia, no hitting, kicking, or beating, no life threat, and no report of being insulted or humiliated more often than rarely, either as a child or an adult.

\section{Assessment of rectal sensation thresholds}

Rectal sensory thresholds were assessed using an electronic barostat. The Chapel Hill site used the Synectics Visceral Stimulator (Synectics Medtronics Co., Shoreham, Minnesota, USA), and the Toronto site used the G\&J Barostat (G\&J Electronics, Toronto, Ontario, Canada). These barostats were programmed to provide distensions at controlled rates of inflation $(38 \mathrm{ml} / \mathrm{s}$ ), duration (30 second inflation alternating with 30 second deflation), and pressure. The catheter consisted of a polyethylene multilumen tube $5 \mathrm{~mm}$ in outside diameter to the end of which a single use thin walled plastic bag (Mui Scientific, Mississippi, Ontario, Canada) was tied at each end so that the length of the balloon was $10 \mathrm{~cm}$. Independent lumens were used to inflate the bag and to measure pressure within the bag. ${ }^{21}$ The plastic bag was infinitely compliant up to its maximum capacity of $600 \mathrm{ml}$. The bag had a maximum diameter of $15 \mathrm{~cm}$ and was hexagonal in shape.

Subjects were asked to give a subjective rating of the intensity of their sensations following each distension using a six point scale $(0=$ no sensation to $5=$ intense sensation $)$. Subjects rated the intensity of pain and the intensity of urgency to defecate separately at the end of each distension.

Rectal sensory thresholds were determined by ascending method of limit (AML) and by the tracking technique (TT). ${ }^{21}$ In the AML, successive phasic balloon distensions were each $4 \mathrm{~mm} \mathrm{Hg}$ greater than the previous distension until the patient first reported a sensation of moderate pain on a six point rating scale. This defined the AML threshold for pain. The AML threshold for urge to defecate was the lowest pressure and volume of distension that elicited a report of moderate urge.

The TT involved 20 unpredictable phasic balloon distensions which tracked the subject's pain threshold. Following each trial on which the subject reported pain/discomfort of moderate intensity or greater (beginning at the threshold for moderate pain, as defined by AML), the next trial was either at the same intensity or $2 \mathrm{~mm} \mathrm{Hg}$ lower in intensity. If the subject rated the trial less than moderate in intensity, the next trial was either the same or $2 \mathrm{~mm} \mathrm{Hg}$ greater. ${ }^{21}$ The pain threshold by TT was defined as the mean volume and pressure for all trials rated at least moderately painful.

The sensory threshold throughout the study was defined as "at least moderately painful" to assure that subjects were reporting pain and not a vague discomfort.

\section{Assessment of clinical symptom variables}

Abdominal pain was assessed using three separate measures: abdominal pain immediately before the physiological test, averaged daily pain for the two preceding weeks, and abdominal pain for the previous 24 hours. The first two variables were rated on a visual analogue scale (VAS) and the latter on an ordinal scale. A VAS was also used to assess anxiety immediately prior to physiological testing.

\section{Data analysis}

Preliminary analysis revealed that the groups differed in age, education, and marital status (table 1). Consequently, the groups were compared by analysis of covariance with statistical adjustment for age and years of education. No adjustment was made for marital status because the groups did not differ with respect to the proportion who were married or co-habiting; they differed only in the proportion who had never married (more non-abused) and the proportion who were divorced (more severely abused patients). The alpha level used for all statistical tests was 0.05 .

The two sites used different barostats, and these machines gave slightly different values because of technical differences in how they inflated the bag. These between site differences were counterbalanced by testing approximately equal numbers of severely abused and non-abused patients at each site. However, to insure that between site differences did not influence the outcome of the study, we recomputed the analysis of covariance after transforming the pain threshold data at each site into z scores which could be pooled across sites. The formula for $\mathrm{z}$ score transformation was to subtract the mean of all observations at a site from each individual observation and divide this difference by the standard deviation for all values at the site. This yields a mean of 0 and a standard deviation of 1 for all patients tested at each site, but it preserves individual differences among the patients tested at the site.

A multiple linear regression model was used to determine whether abuse is a significant predictor of sensory thresholds after adjusting for demographic variables that were found to be different between the abused and non-abused groups.

Separate ANOVA analyses were used to explore whether the type of abuse experienced (sexual, physical, and both sexual and physical) influences sensory thresholds (pain threshold by AML, pain threshold by TT, and urge sensation by AML).

\section{RESULTS}

From a total pool of 278 patients with IBS, 74 (46\%) met criteria for severe sexual/physical abuse and 85 reported no history of abuse. The remainder (that is, those who reported mild to moderate severity of abuse experiences) were not included in the analyses.

\section{Demographic characteristics of the sample}

Table 1 shows the demographic characteristics of the patients studied. Severely abused patients were approximately five years older than non-abused patients, and they had discontinued school approximately one year earlier. There were no significant differences in the racial composition of the groups. However, there were differences in marital status: although comparable proportions of both groups were married or co-habiting, a smaller proportion of the severely abused group were single (possibly related to older average age), and a larger proportion of the abused group were divorced.

\section{Rectal pain thresholds}

IBS patients with a history of severe abuse had significantly higher rectal pain thresholds, as measured by the pressure required to produce the first report of pain (AML threshold; $F$ $(1,111)=6.06 ; p=0.015)$. When pain threshold was measured by the pressure required to consistently produce a report of moderate pain on the tracking task, severely abused IBS patients also had significantly higher pain thresholds $(\mathrm{F}(\mathrm{l}, 109)=5.21 ; \mathrm{p}=0.024)$. These data are shown in fig 1. Patients with a history of severe abuse also reported a significantly higher threshold for experiencing a 


\begin{tabular}{|c|c|c|c|}
\hline & $\begin{array}{l}\text { No abuse } \\
(\mathrm{n}=85)\end{array}$ & $\begin{array}{l}\text { Severe abuse } \\
(n=74)\end{array}$ & Statistical test ( $p$ value) \\
\hline Age $(y)$ & $34.8(11.8)$ & $39.5(11.1)$ & $T(157)=2.59,(p=0.011)$ \\
\hline $\begin{array}{l}\text { Education (y) } \\
\text { Race (\%) }\end{array}$ & $15.3(2.8)$ & $14.3(2.6)$ & $\begin{array}{l}\mathrm{T}(155)=2.36,(p=0.019) \\
\chi^{2}(4)=0.259,(p=0.259)\end{array}$ \\
\hline Caucasian & 91.8 & 85.1 & \\
\hline African American & 5.9 & 8.1 & \\
\hline Other minority & 2.4 & 6.8 & \\
\hline Marital status (\%) & & & $\chi^{2}(3)=8.15,(p=0.043)$ \\
\hline Married or co-habiting & 47.6 & 43.2 & \\
\hline Single & 40.5 & 28.4 & \\
\hline Divorced & 11.9 & 25.7 & \\
\hline Widowed & 0 & 2.7 & \\
\hline
\end{tabular}

sensation of urgency to defecate $(F \quad(1,113)=11.23$; $\mathrm{p}=0.001$ ), as shown in fig 2 .

To insure that the differences between severely abused and non-abused patients shown in figs 1 and 2 were not confounded by between site differences, pressure and volume data were transformed to $\mathrm{z}$ scores and the analyses repeated. For first report of moderate pain $(\mathrm{F} \quad(1,111)=4.12$; $\mathrm{p}=0.045$ ) and first report of urge to defecate ( $\mathrm{F}$ ( 1 , $113)=8.72 ; p=0.004)$, differences between severely abused and non-abused patients remained significant. The tracking pain threshold showed a strong trend, consistent with the previous analysis, but it failed to reach statistical significance $(\mathrm{F}(1,109)=3.91 ; \mathrm{p}=0.059)$.

\section{Secondary analysis}

As women in the abused group were older, more likely to be divorced, and received less years of education than nonabused women, we further explored these group differences by multiple linear regression. After adjusting for the effect of age, marital status, and education, abuse history had higher rectal pain thresholds by tracking ( $\beta$ estimate $=4.05$, $p=0.05)$ and AML $(\beta$ estimate $=4.41, p=0.02)$, and higher urge sensation thresholds by AML $(\beta$ estimate $=4.58$, $p=0.01)$. If we were to interpret these secondary analysis as primary tests, a Bonferroni adjustment for multiple comparison and a p value of $<0.017$ would be required for significance.

Furthermore, in order to explore whether higher pain thresholds were related to the type of abuse experienced, we divided the severely abused group into three subgroups according to the nature of the abuse: sexual $(n=47)$, physical $(n=12)$, both sexual and physical $(n=14)$. Using ANOVA analysis, we looked separately at the three variables

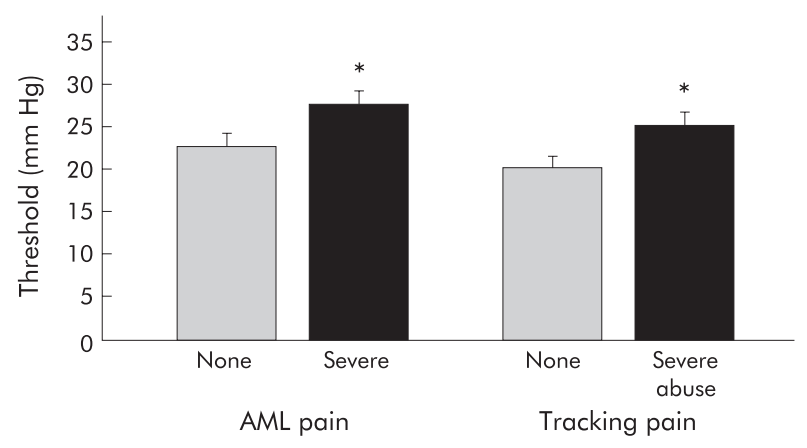

Figure 1 Association of sexual abuse history with rectal pain thresholds. Patients with irritable bowel syndrome with a history of severe abuse had significantly higher rectal pain thresholds, as measured by the ascending method of limit (AML) and by the tracking technique. ${ }^{*} \mathrm{p}<0.03$.

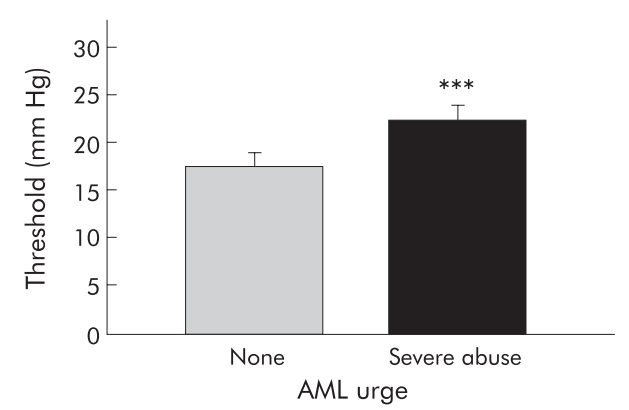

Figure 2 Association of sexual abuse history with urge sensation thresholds. Patients with irritable bowel syndrome with a history of severe abuse had significantly higher urge sensation thresholds, as measured by the ascending method of limit (AML). ${ }^{* *} p<0.001$.

of interest (that is, pain threshold by AML, pain threshold by TT, and urge sensation by AML) across the three subgroups. There were no significant differences between the three groups for any sensory parameter; however, for tracking pain thresholds, group means were 27.8 (SD 14) for sexual abuse, 24.7 (12) for physical abuse, and 20.1 (8) for both types of abuse $(\mathrm{p}=0.18)$.

\section{Clinical symptom variables}

Table 2 shows the relationship between abuse and clinical variables. No significant differences were found for any of the variables investigated (that is, average of 14 daily abdominal pain ratings for the two weeks preceding the physiological test, VAS rating of current pain immediately before the physiological test, ordinal scale rating of abdominal pain for the previous 24 hours, or VAS rating of anxiety immediately prior to the physiological test).

\section{DISCUSSION}

Epidemiological studies have suggested a link between a history of abuse and a diagnosis of more severe IBS ${ }^{15}{ }^{16}$; IBS patients with a history of abuse have more severe illness and poorer health outcomes. ${ }^{9}$ Several possible mechanisms have been suggested to explain this association ${ }^{8}$ : (1) a history of abuse may have a sensitising peripheral effect that contributes to heightened visceral sensitivity (that is, peripheral disordered visceral function); or (2) abuse may have a centrally mediated effect on modulation of the conscious perception of visceral signals and/or altered affective or behavioural responses to afferent visceral signals, thus leading to more severe symptoms and adverse clinical outcome. Only one reported study has looked at the effect of a history of abuse on visceral (that is, rectal) sensation in patients with IBS. Whitehead and colleagues ${ }^{18}$ examined whether reduced pain thresholds in patients with IBS are 
Table 2 Association of abuse history with clinical variables

\begin{tabular}{llll}
\hline & No abuse & Severe abuse & Statistical test ( $p$ value) \\
\hline $\begin{array}{l}\text { Pain from diary (14 day } \\
\text { average VAS) }\end{array}$ & $38.60(20.11)$ & $41.32(22.46)$ & $F(1,153)=0.70(p=0.403)$ \\
$\begin{array}{l}\text { Current pain (VAS) } \\
\text { Pain for last 24 hours (5 point } \\
\text { ordinal rating) }\end{array}$ & $24.16(24.04)$ & $25.16(21.74)$ & $F(1,119)=0.06(p=0.807)$ \\
$\begin{array}{c}\text { Anxiety prior to the procedure } \\
\text { (VAS) }\end{array}$ & $41.17(24.03)$ & $41.61(24.03)$ & $F(1,118)=0.64(p=0.800)$ \\
\hline $\begin{array}{l}\text { Values are mean (SD)). } \\
\text { VAS, visual analogue scale. }\end{array}$ & & \\
\hline
\end{tabular}

related to psychological factors and/or prior history of sexual abuse. They found that higher pain sensitivity in IBS patients correlated with psychological factors (that is, anxiety and somatisation). However, sexual abuse was not found to be associated with lower pain thresholds. This study included 32 patients with IBS ( 17 with and 15 without abuse history) and 27 controls (13 with and 14 without abuse history). The relatively small number of subjects in this study raised the possibility of lack of power to show differences between the groups (that is, a type II error).

The current study was performed in a larger sample size. Our results show that patients with a history of severe abuse have significantly higher thresholds for rectal pain and urge sensation compared with patients with no history of abuse. In contrast with the findings by Whitehead et al that suggested that abused patients have sensation thresholds no different than non-abused patients, the current study shows that they actually have higher sensation thresholds. These findings were similar by the tracking task and by the ascending method of limits, confirming the pertinence of these finding and reassuring that the multiple distensions did not appear to bias the results.

A possible explanation for this finding is that severely abused patients use a psychological defence mechanism of dissociation in response to a painful rectal stimulus. Previous studies reported higher dissociation scores in subjects with a history of abuse. ${ }^{22}$ This may lead such patients to psychologically "screen out" painful sensations which might possibly trigger memories of prior painful trauma. Regardless of the mechanisms of this phenomenon, our findings do not support the hypothesis that abuse is associated with peripheral visceral hypersensitivity. The results of the secondary analysis further support our finding. After adjusting by linear regression for the effect of age, marital status, and education, patients with a history of severe abuse still had significantly higher urge sensation thresholds, and they tended to have higher rectal pain thresholds.

In this study, we investigated the association between abuse history and rectal sensation. Anal sphincter and pelvic floor dynamics might also be associated with high sensation thresholds. However, these factors were not explored in this study. A few reports in the literature suggest also an association between abuse and motor dysfunction of the pelvic floor and anal sphincter. ${ }^{23}{ }^{24}$ The available data on this issue are very limited and deserve further studies.

Although severely abused patients differed in their tested physiological measures from those with no abuse history, there were no differences between the groups with regard to their ratings of clinical symptoms. These findings are different from previous reports from our group and others showing more severe pain and pain behaviours in patients with a history of abuse. ${ }^{9}$ In those studies the information on abuse was obtained by a comprehensive interview whereas in this study abuse history was ascertained by a self-report screening questionnaire. Although the screening instrument used in this study may be less sensitive as a diagnostic measure for abuse due to reporting bias, it cannot explain the differences in sensory thresholds between the severely abused and non-abused groups in this study.

The association between gastrointestinal illnesses and abuse has been shown in multiple studies from our group and others. These studies have also shown that abuse history is associated with poor adjustment to illness and adverse clinical status, including increased amount of health care seeking. ${ }^{15}$ In a recently published review, Koloski and colleagues $^{25}$ reported that the severity of symptoms in patients with IBS and non-ulcer dyspepsia does not fully explain consulting behaviour. However, psychosocial factors (including a history of abuse) were found to be more strongly associated with health care seeking. Therefore, it seems likely that the association between abuse and pain behaviours in IBS patients might relate to the central effect of abuse in modulating the affective and behavioural responses to afferent visceral signals rather than to increased peripheral visceral sensation.

Further support for a possible central modulating role of abuse on affective and behavioural responses may come from recently reported studies using brain imaging techniques. These studies have shown morphological (that is, decreased hippocampal mass) ${ }^{26} 27$ and functional (that is, reduced activation of anterior cingulate cortex $)^{28}$ abnormalities in subjects with abuse history. We have recently examined the effect of abuse in patients with IBS using PET $^{29}$ and fMRI. ${ }^{30-32}$ We found that a history of abuse modulates the anterior cingulate cortex activation, a region involved in the motivational and affective reactions to painful stimulation. Furthermore, these alterations in cingulate cortex activity are associated with pain reports and correlate with psychosocial distress. ${ }^{33} 34$

Our study provides new and somewhat surprising sensory threshold data. However, it does not provide a mechanistic explanation regarding the possible causes for these findings. Further studies are needed to look at the effects of abuse history (and other psychosocial factors) on physiological functioning at different levels of the brain-gut axis, and their relevance to symptom severity, pain behaviours, and outcome. Better understanding of these psychological-physiological correlates will help to clarify theories about the mechanisms underlying the development of symptoms in IBS and other functional gastrointestinal disorders, and will contribute to improving our treatment of these patients.

\section{ACKNOWLEDGEMENTS}

The authors would like to thank Ms Carolyn Morris and Ms Birva Shah for their help in preparing and editing this manuscript.

\section{Authors' affiliations}

Y Ringel, W E Whitehead, Y Hu, H Jia, S I Bangdiwala, D A Drossman, Department of Medicine, Division of Digestive Diseases and Nutrition, and the UNC Center for Functional GI and Motility Disorders, University of North Carolina at Chapel Hill, North Carolina, USA 
B B Toner, Centre for Addiction and Mental Health, Department of Psychiatry, University of Toronto, Ontario, Canada

NE Diamant, Division of Gastroenterology, University of Toronto, Ontario, Canada

\section{REFERENCES}

1 Mertz H, Naliboff B, Munakata J, et al. Altered rectal perception is a biological marker of patients with irritable bowel syndrome. Gastroenterol 1995; 109:40-52.

2 Whitehead WE, Palsson OS. Is rectal pain sensitivity a biological marker for irritable bowel sydrome: Psychological influences on pain perception. Gastroenterology 1998;115:1-10.

3 Mayer EA, Gebhart GF. Basic and clinical aspects of visceral hyperalgesia. Gastroenterology 1994;107:271-93.

4 Rao SS. Visceral hyperalgesia: The key for unrevealing functional gastrointestinal disorders. Dig Dis 1996;14:271-5.

5 Mayer EA, Raybould HE. Role of visceral afferent mechanisms in functional bowel disorders. Gastroenterology 1990;99:1688-704

6 Whitehead WE, Drossman DA, Diamant N, et al. Correlation of changes in clinical pain and abnormal stools with changes in visceral pain thresholds following treatment. Gastroenterology 2000;1 18(suppl 2):A128.

7 Ringel Y, Sperber AD, Drossman DA. Irritable bowel syndrome. Annu Rev Med 2001;52:319-38.

8 Drossman DA, Creed FH, Olden KW, et al. Psychosocial aspects of the functional gastrointestinal disorders. In: Drossman DA, Corazziari E, Talley NJ, et al. Rome II, The functional gastrointestinal disorders: Diagnosis, pathophysiology and treatment; A multinational consensus, 2nd edn. McLean, VA: Degnon and Associates, 2000:157-245.

9 Drossman DA, Li Z, Leserman J, et al. Health status by gastrointestinal diagnosis and abuse history. Gastroenterology 1996;1 10:999-1007.

10 Drossman DA, Whitehead WE, Toner BB, et al. What determines severity among patients with painful functional bowel disorders? Am J Gastroenterol 2000;95:974-80.

11 Drossman DA. Do psychosocial factors define symptom severity and patient status? Am J Med 1999; 107:41S-50.

12 Drossman DA, Camilleri M, Mayer EA, et al. AGA technical review on irritable bowel syndrome. Gastroenterology 2002;123:2108-31.

13 Talley NJ, Fett SL, Zinsmeister AR. Self-reported abuse and gastrointestinal disease in outpatients: Association with irritable bowel-type symptoms. Am J Gastroenterol 1995;90:366-71.

14 Walker EA, Gelfand AN, Gelfand MD, et al. Psychiatric diagnoses, sexual and physical victimization, and disability in patients with irritable bowel syndrome or inflammatory bowel disease. Psychol Med 1995;25:1259-67.

15 Drossman DA, Talley NJ, Leserman J, et al. Sexual and physical abuse and gastrointestinal illness: Review and recommendations. Ann Intern Med 1995; 123:782-94.

16 Drossman DA. Irritable bowel syndrome and sexual/physical abuse history. Eur J Gastroenterol Hepatol 1997;9:327-30.

17 Drossman DA. Sexual and physical abuse and gastrointestinal illness. Scand J Gastroenterol Suppl 1995;208:90-6.
18 Whitehead WE, Crowell MD, Davidoff AL, et al. Pain from rectal distension in women with irritable bowel syndrome: Relationship to sexual abuse. Dig Dis Sci 1997;42:796-804.

19 Drossman DA, Li Z, Toner BB, et al. Functional bowel disorders: A multicenter comparison of health status, and development of illness severity index. Dig Dis Sci 1995;40:986-95.

20 Leserman J, Li Z, Drossman DA, et al. Impact of sexual and physical abuse dimensions on health status: Development of an abuse severity measure. Psychosom Med 1997:59:152-60.

21 Whitehead WE, Delvaux M, Working Team. Standardization of procedures for testing smooth muscle tone and sensory thresholds in the gastrointestinal tract. Dig Dis Sci 1994;42:223-41.

22 Farley M, Keaney JC. Physical symptoms, somatization, and dissociation in women survivors of childhood sexual assault. Women Health 1997; 25:33-45.

23 Leroi AM, Berkelmans I, Denis P, et al. Anismus as a marker of sexual abuse. Consequences of abuse on anorectal motility. Dig Dis Sci 1995;40:1411-16.

24 Devroede G. Early life abuses in the past history of patients with gastrointestinal tract and pelvic floor dysfunctions. Prog Brain Res 2000;122:131-55.

25 Koloski NA, Talley NJ, Boyce PM. Predictors of health care seeking for irritable bowel syndrome and nonulcer dyspepsia: A critical review of the literature on symptom and psychosocial factors. Am J Gastroenterol 2001;96:1340-9.

26 Bremner JD, Randall P, Vermetten E, et al. Magnetic resonance imagingbased measurement of hippocampal volume in posttraumatic stress disorder related to childhood physical and sexual abuse-A preliminary report. Biol Psychiatry 1997;41:23-32.

27 Stein MB, Koverola C, Hanna C, et al. Hippocampal volume in women victimized by childhood sexual abuse. Psychol Med 1997;27:951-9.

28 Semple WE, Goyer P, McCormick R, et al. Preliminary report: brain blood flow using PET in patients with posttraumatic stress disorder and substanceabuse histories. Biol Psychiatry 1993;34:115-18.

29 Ringel Y, Drossman DA, Turkington TG, et al. Regional brain activation in response to rectal distention in patients with irritable bowel syndrome and the effect of a history of abuse. Dig Dis Sci 2003;48:1774-81.

30 Ringel $Y$, Drossman DA, Liu $\mathrm{H}$, et al. Anterior shift of cingulate activity with painful rectal distension in subjects with sexual/physical abuse. Gastroenterology 2002;122:A31 1

31 Ringel Y, Drossman DA, Liu $\mathrm{H}$, et al. FMRI of cingulate activation to painful rectal distension in IBS and sexual/physical abuse. Gastroenterology 2002;122:A311.

32 Ringel Y, Drossman DA, Leserman J, et al. IBS diagnosis and a history of abuse have synergistic effect on the perigenual cingulate activation in response to rectal distention. Gastroenterology 2003;124:A531.

33 Drossman DA, Ringel $Y$, Vogt $B$, et al. Alterations of brain activity associated with resolution of emotional distress and pain in a case of severe IBS. Gastroenterology, 124:754-61.

34 Ringel Y, Drossman DA, Leserman J, et al. Association of anterior cingulate cortex $(\mathrm{ACC})$ activation with psychosocial distress and pain reports. Gastroenterology 2003;124:A97. 